\title{
THE LEADING IDEAL OF A COMPLETE INTERSECTION OF HEIGHT TWO
}

\author{
SHIRO GOTO, WILLIAM HEINZER, AND MEE-KYOUNG KIM
}

\begin{abstract}
Let $(S, \mathfrak{n})$ be a Noetherian local ring and let $I=(f, g)$ be an ideal in $S$ generated by a regular sequence $f, g$ of length two. Assume that the associated graded ring $\operatorname{gr}_{\mathfrak{n}}(S)$ of $S$ with respect to $\mathfrak{n}$ is a UFD. We examine generators of the leading form ideal $I^{*}$ of $I$ in $\operatorname{gr}_{\mathfrak{n}}(S)$ and prove that $I^{*}$ is a perfect ideal of $\operatorname{gr}_{\mathfrak{n}}(S)$, if $I^{*}$ is 3-generated. Thus, in this case, letting $R=S / I$ and $\mathfrak{m}=\mathfrak{n} / I$, if $\operatorname{gr}_{\mathfrak{n}}(S)$ is Cohen-Macaulay, then $\operatorname{gr}_{\mathfrak{m}}(R)=\operatorname{gr}_{\mathfrak{n}}(S) / I^{*}$ is Cohen-Macaulay. As an application, we prove that if $(R, \mathfrak{m})$ is a one-dimensional Gorenstein local ring of embedding dimension 3 , then $\operatorname{gr}_{\mathfrak{m}}(R)$ is Cohen-Macaulay if the reduction number of $\mathfrak{m}$ is at most 4 .
\end{abstract}

\section{INTRODUCTION}

Setting 1.1. Let $(S, \mathfrak{n})$ be a Noetherian local ring and let $I=(f, g)$ be an ideal in $S$ generated by a regular sequence $f, g$ of length two. Let $R=S / I$ and $\mathfrak{m}=\mathfrak{n} / I$. Let

$$
\mathrm{R}^{\prime}(\mathfrak{n})=\sum_{i \in \mathbb{Z}} \mathfrak{n}^{i} t^{i} \subseteq S\left[t, t^{-1}\right] \quad \text { and } \quad \mathrm{R}^{\prime}(\mathfrak{m})=\sum_{i \in \mathbb{Z}} \mathfrak{m}^{i} t^{i} \subseteq R\left[t, t^{-1}\right]
$$

denote the extended Rees algebras of $\mathfrak{n}$ and $\mathfrak{m}$ respectively, where $t$ is an indeterminate. Let

$$
\operatorname{gr}_{\mathfrak{n}}(S)=\mathrm{R}^{\prime}(\mathfrak{n}) / t^{-1} \mathrm{R}^{\prime}(\mathfrak{n}) \quad \text { and } \quad \operatorname{gr}_{\mathfrak{m}}(R)=\mathrm{R}^{\prime}(\mathfrak{m}) / t^{-1} \mathrm{R}^{\prime}(\mathfrak{m})
$$

Then the canonical map $S \rightarrow R$ induces the homomorphism $\varphi: \operatorname{gr}_{\mathfrak{n}}(S) \rightarrow \operatorname{gr}_{\mathfrak{m}}(R)$ of the associated graded rings. We put

$$
I^{*}=\operatorname{Ker}\left(\operatorname{gr}_{\mathfrak{m}}(S) \stackrel{\varphi}{\rightarrow} \operatorname{gr}_{\mathfrak{m}}(R)\right) .
$$

Then the ideal $I^{*}$ is generated by the initial forms of elements of $I$ and $\operatorname{gr}_{\mathfrak{m}}(R) \cong$ $\operatorname{gr}_{\mathfrak{n}}(S) / I^{*}$. We assume that $G=\operatorname{gr}_{\mathfrak{n}}(S)$ is a UFD. Hence $\operatorname{ht}_{G} I^{*}=\operatorname{grade}_{G} I^{*}=2$.

Date: July 29, 2005.

1991 Mathematics Subject Classification. Primary: 13A30, 13C05; Secondary: 13E05, 13 H15.

Key words and phrases. ideal of initial forms, associated graded ring, multiplicity, reduction number, Gorenstein ring, Cohen-Macaulay ring.

Shiro Goto is supported by the Grant-in-Aid for Scientific Researches in Japan $(\mathrm{C}(2)$, No.1364044).

Mee-Kyoung Kim is supported by the Korea Science and Engineering Foundation (R04-2003000-10113-0). 
We are interested in determining generators for $I^{*}$ and thereby obtaining conditions in order that $\operatorname{gr}_{\mathfrak{m}}(R)$ be Cohen-Macaulay. The goal of the paper is to prove Theorem 1.2, the proof of which is given in Section 2 .

Theorem 1.2. Assume notation as in Setting 1.1, so, in particular, $\operatorname{gr}_{\mathfrak{n}}(S)$ is a $U F D$. If $I^{*}$ is 3-generated, then $I^{*}$ is a perfect ideal of $\operatorname{gr}_{\mathfrak{n}}(S)$. Therefore if $\operatorname{gr}_{\mathfrak{n}}(S)$ is Cohen-Macaulay, then $\operatorname{gr}_{\mathfrak{m}}(R)=\operatorname{gr}_{\mathfrak{n}}(S) / I^{*}$ is Cohen-Macaulay.

As an immediate corollary to Theorem 1.2, we have

Corollary 1.3. With notation as in Setting 1.1, if $(S, \mathfrak{n})$ is a regular local ring and $I^{*}$ is 3-generated, then $\operatorname{gr}_{\mathfrak{m}}(R)$ is Cohen-Macaulay.

In Section 3 we discuss some consequences of Theorem 1.2.

Notation 1.4. Let $G=\operatorname{gr}_{\mathfrak{n}}(S)$. For each $f \in S$ let $o(f)=\sup \left\{i \in \mathbb{Z} \mid f \in \mathfrak{n}^{i}\right\}$, the order of $f$. We put

$$
f^{*}= \begin{cases}\overline{f t^{i}} & \text { if } f \neq 0 \text { and } i=o(f) \\ 0 & \text { if } f=0\end{cases}
$$

and call it the initial form of $f$, where $\overline{f t^{i}}$ denotes the image in $G$ of $f t^{i} \in \mathfrak{n}^{i} t^{i}$ in $\mathrm{R}^{\prime}(\mathfrak{n})$. Then for all $f, g \in S$ we have

$$
\begin{aligned}
& o(f g)=o(f)+o(g), \quad(f g)^{*}=f^{*} g^{*}, \\
& o(f+g) \geq \min \{o(f), o(g)\}, \text { and } \\
& o(f+g)=\min \{o(f), o(g)\} \text { if } o(f) \neq o(g) .
\end{aligned}
$$

With this notation the following two simple examples illustrate the situation we are considering. In both examples we let $S=k[[x, y, z]]$ be the formal power series ring in the three variables $x, y, z$ over a field $k$.

Example 1.5. Let $R=k\left[\left[w^{5}, w^{6}, w^{9}\right]\right]$ be the subring of the formal power series ring $k[[w]]$ and define the homomorphism $\phi: S \rightarrow R$ of $k$-algebras by $\phi(x)=w^{5}$, $\phi(y)=w^{6}$, and $\phi(z)=w^{9}$. Then the ideal $I=\operatorname{Ker} \phi$ is generated by $f=z^{2}-y^{3}$ and $g=z y-x^{3}$, whence $R$ is a complete intersection of dimension one. We have $\operatorname{gr}_{\mathfrak{n}}(S)=k\left[x^{*}, y^{*}, z^{*}\right], f^{*}=z^{* 2}$, and $g^{*}=z^{*} y^{*}$. Let $h=y f-z g=z x^{3}-y^{4}$. Then $h^{*}=z^{*} x^{* 3}-y^{* 4}$. Let

$$
J=\left(f^{*}, g^{*}, h^{*}\right)=\left(z^{* 2}, z^{*} y^{*}, z^{*} x^{* 3}-y^{* 4}\right) \subseteq I^{*} .
$$


Then the Hilbert series of the graded $\operatorname{ring} \operatorname{gr}_{\mathfrak{n}}(S) / J$ is

$$
\frac{1+2 t+t^{2}+t^{3}}{1-t}=1+3 t+4 t^{2}+5 t^{3}+5 t^{4}+\cdots+5 t^{n}+\cdots
$$

and these values are the same as those in the Hilbert series of $\operatorname{gr}_{\mathfrak{m}}(R)=\operatorname{gr}_{\mathfrak{n}}(S) / I^{*}$, so that $J=I^{*}$. The reduction number of $\mathfrak{m}=\left(w^{5}, w^{6}, w^{9}\right)$ with respect to the

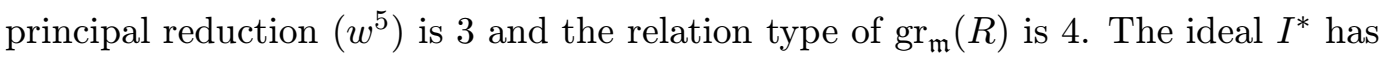
grade 2 and is generated by the $2 \times 2$ minors of the following matrix

$$
\left[\begin{array}{ccc}
y^{*} & z^{*} & 0 \\
-x^{* 3} & -y^{* 3} & z^{*}
\end{array}\right]
$$

Hence, by the theorem of Hilbert-Burch [BH, Theorem 1.4.17], $I^{*}$ is a perfect ideal and $\operatorname{gr}_{\mathfrak{n}}(S) / I^{*}=\operatorname{gr}_{\mathfrak{m}}(R)$ is a Cohen-Macaulay ring.

Example 1.6. Let $R=k\left[\left[w^{6}, w^{7}, w^{15}\right]\right]$ be the subring of the formal power series ring $k[[w]]$ and consider the homomorphism $\phi: S \rightarrow R$ of $k$-algebras defined by $\phi(x)=w^{6}, \phi(y)=w^{7}$, and $\phi(z)=w^{15}$. Then $I=\operatorname{Ker} \phi$ is generated by $f=z^{2}-x^{5}$ and $g=z x-y^{3}$, whence $R$ is a complete intersection of dimension one. We have $\operatorname{gr}_{\mathfrak{n}}(S)=k\left[x^{*}, y^{*}, z^{*}\right], f^{*}=z^{* 2}$, and $g^{*}=z^{*} x^{*}$. Let $h=x f-z g=z y^{3}-x^{6}$. Then $h^{*}=z^{*} y^{* 3}$ and $\left(f^{*}, g^{*}, h^{*}\right)=\left(z^{* 2}, z^{*} x^{*}, z^{*} y^{* 3}\right) \subsetneq I^{*}$. The inclusion is strict, since $\mathrm{ht}_{\mathrm{gr}_{\mathfrak{n}}(S)} I^{*}=2$ and $z^{*}$ is a common factor of $f^{*}, g^{*}$, and $h^{*}$. We have $o(f)=o(g)=2$ and $o(h)=4$. Let $h_{1}=x h-y^{3} g=y^{6}-z^{7} \in I$. Then $h_{1}^{*}=y^{* 6}$. We put

$$
J=\left(z^{* 2}, z^{*} x^{*}, z^{*} y^{* 3}, y^{* 6}\right) \subseteq I^{*} .
$$

Then the Hilbert series of $\operatorname{gr}_{\mathfrak{n}}(S) / J$ is given by

$$
\frac{1+2 t+t^{2}+t^{3}+t^{5}}{1-t}=1+3 t+4 t^{2}+5 t^{3}+5 t^{4}+6 t^{5}+\cdots+6 t^{n}+\cdots
$$

and these values are the same as those in the Hilbert series of $\operatorname{gr}_{\mathfrak{m}}(R)=\operatorname{gr}_{\mathfrak{n}}(S) / I^{*}$, so that $J=I^{*}$. The reduction number of $\mathfrak{m}=\left(w^{6}, w^{7}, w^{15}\right)$ with respect to the

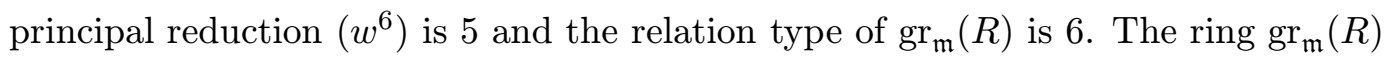
is not Cohen-Macaulay. This is implied by the gap in the numerator of the Hilbert series, and can be deduced also from the fact that the ideal $I^{*}$ has radical $\left(y^{*}, z^{*}\right)$ and the ideal $I^{*}: z^{*}$ is primary with $\sqrt{I^{*}: z^{*}}=\left(x^{*}, y^{*}, z^{*}\right)$. 


\section{Proof of Theorem 1.2}

The purpose of this section is to prove Theorem 1.2. We assume notation as in Setting 1.1. Let $G=\operatorname{gr}_{\mathfrak{n}}(S)$ and $J=I^{*}$. We choose $f, g \in S$ so that $I=(f, g)$ with $a=o(f) \leq b=o(g)$. Without loss of generality we may assume that $f^{*} \notin N J$ and $g^{*} \notin N J+\left(f^{*}\right)$, where $N=G_{+}$. Hence the elements $f^{*}, g^{*}$ form part of a minimal system of homogeneous generators of $J$. Notice that if $\operatorname{ht}_{G}\left(f^{*}, g^{*}\right)=2$, then the sequence $f^{*}, g^{*}$ is $G$-regular whence $J=\left(f^{*}, g^{*}\right)$. In what follows we assume that

$$
\operatorname{ht}_{G}\left(f^{*}, g^{*}\right)=1 \text {. }
$$

Let $D=\operatorname{GCD}\left(f^{*}, g^{*}\right)$ and write $f^{*}=\xi D, g^{*}=\eta D$, where $D, \xi, \eta$ are homogeneous elements of $G$ with degree $d>0, a-d$, and $b-d$, respectively. Then $\{\xi, \eta\}$ is a $G$-regular sequence.

We begin with Lemma 2.1 which gives some information about homogeneous elements of $J$ that are not in the ideal $\left(f^{*}, g^{*}\right)$.

Lemma 2.1. Let $\alpha, \beta \in S$ and $h=\alpha f+\beta g$. Assume that $h^{*} \notin\left(f^{*}, g^{*}\right)$. Then

(1) $o(\alpha f)=o(\beta g)<o(h)$.

(2) $o(\alpha)+a=o(\beta)+b, o(\alpha) \geq b-d$, and $o(\beta) \geq a-d$.

(3) $\alpha^{*} \xi+\beta^{*} \eta=0$.

Proof. We have $o(h) \geq \min \{o(\alpha f), o(\beta g)\}$. If $o(\alpha f)<o(\beta g)$, then $o(h)=o(\alpha f)$ and $h^{*}=\alpha^{*} f^{*} \in\left(f^{*}\right)$, which is impossible. We similarly have $o(\alpha f)=o(\beta g)$. Hence $o(h)>o(\alpha f)=o(\beta g)$, because $h^{*} \notin\left(f^{*}, g^{*}\right)$. Thus $\alpha^{*} f^{*}+\beta^{*} g^{*}=\left(\alpha^{*} \xi+\beta^{*} \eta\right) D=0$ whence $\alpha^{*} \xi+\beta^{*} \eta=0$. Therefore, since the sequence $\xi, \eta$ is $G$-regular, we get $\alpha^{*}=$ $-\varphi \eta$ and $\beta^{*}=\varphi \xi$ for some homogeneous element $\varphi$ of $G$. Thus $o(\alpha)=\operatorname{deg} \varphi+(b-d)$ and $o(\beta)=\operatorname{deg} \varphi+(a-d)$, so that $o(\alpha)+a=o(\beta)+b, o(\alpha) \geq b-d$, and $o(\beta) \geq a-d$, as was claimed.

The existence of a third generator of the leading ideal $J$ of a certain form is guaranteed by Proposition 2.2.

Proposition 2.2. Assume that the local ring $S$ is $\mathfrak{n}$-adically complete. Then there exist elements $\alpha, \beta$ of $S$ such that $o(\alpha)=b-d, o(\beta)=a-d$, and $(\alpha f+\beta g)^{*} \notin$ $\left(f^{*}, g^{*}\right)$. 
Proof. Assume the contrary. Let $f_{0}, g_{0} \in S$ with $o\left(f_{0}\right)=a-d$ and $o\left(g_{0}\right)=b-d$ such that $\xi=f_{0}^{*}$ and $\eta=g_{0}^{*}$. We are going to construct two sequences $\left\{f_{i}\right\}_{i=0,1,2, \ldots}$ and $\left\{g_{i}\right\}_{i=0,1,2, \ldots}$ of elements in $S$ which satisfy the following conditions: Let $h_{i}=$ $\left(-\sum_{k=0}^{i} g_{k}\right) f+\left(\sum_{k=0}^{i} f_{k}\right) g$ for each $i \geq 0$. Then

(1) $h_{i} \neq 0$,

(2) $o\left(h_{i}\right)<o\left(h_{i+1}\right)$,

(3) $o\left(h_{i}\right)-b \leq o\left(f_{i+1}\right)$ and $o\left(h_{i}\right)-a \leq o\left(g_{i+1}\right)$

for all $i \geq 0$.

To construct the sequences, firstly we put $h_{0}=\left(-g_{0}\right) f+f_{0} g$. Then $o\left(f_{0}\right)=a-d$ and $o\left(g_{0}\right)=b-d$. We notice $h_{0} \neq 0$, because $b-d=o\left(g_{0}\right)<o(g)=b$ (recall that $f, g$ is a regular sequence). Hence $h_{0}^{*} \in\left(f^{*}, g^{*}\right)$ by our assumption. We write $h_{0}^{*}=f^{*} \varphi+g^{*} \psi$ with $\varphi \in G_{o\left(h_{0}\right)-a}$ and $\psi \in G_{o\left(h_{0}\right)-b}$. Let $\varphi=\overline{g_{1} t^{o\left(h_{0}\right)-a}}$ and $\psi=\overline{\left(-f_{1}\right) t^{o\left(h_{0}\right)-b}}$ with $g_{1} \in \mathfrak{n}^{o\left(h_{0}\right)-a}$ and $f_{1} \in \mathfrak{n}^{o\left(h_{0}\right)-b}$. Then $h_{0}=g_{1} f+\left(-f_{1}\right) g+h_{1}$ for some $h_{1} \in \mathfrak{n}^{o\left(h_{0}\right)+1}$; hence

$$
h_{1}=\left[-\left(g_{0}+g_{1}\right)\right] f+\left(f_{0}+f_{1}\right) g,
$$

where $o\left(f_{1}\right) \geq o\left(h_{0}\right)-b, o\left(g_{1}\right) \geq o\left(h_{0}\right)-a$, and $o\left(h_{1}\right)>o\left(h_{0}\right)$. Because

$$
\begin{aligned}
\overline{h_{0} t^{a+b-d}} & =\overline{\left(-g_{0}\right) t^{b-d}} \cdot \overline{f t^{a}}+\overline{f_{0} t^{a-d}} \cdot \overline{g t^{b}} \\
& =\left(-\eta f^{*}\right)+\xi g^{*} \\
& =(-\eta \cdot \xi D)+\xi(\eta D) \\
& =0
\end{aligned}
$$

we get $o\left(h_{0}\right)>a+b-d$, so that $o\left(f_{1}\right) \geq o\left(h_{0}\right)-b>a-d$ and $o\left(g_{1}\right) \geq o\left(h_{0}\right)>b-d$. Thus $o\left(g_{0}+g_{1}\right)=o\left(g_{0}\right)=b-d<b$ and $o\left(f_{0}+f_{1}\right)=o\left(f_{0}\right)=a-d<a$, whence $h_{1}=\left[-\left(g_{0}+g_{1}\right)\right] f+\left(f_{0}+f_{1}\right) g \neq 0$. Repeating this procedure, we get the required sequences $\left\{f_{i}\right\}_{i=0,1,2, \ldots}$ and $\left\{g_{i}\right\}_{i=0,1,2, \ldots}$ of elements in $S$.

Now let $\alpha=-\sum_{k=0}^{\infty} g_{k}$ and $\beta=\sum_{k=0}^{\infty} f_{k}$. We then have

$$
\begin{aligned}
\alpha f+\beta g & =\sum_{k=0}^{\infty}\left[\left(-g_{k}\right) f+f_{k} g\right] \\
& =\lim _{i \rightarrow \infty}\left[\left(-\sum_{k=0}^{i} g_{k}\right) f+\left(\sum_{k=0}^{i} f_{k}\right) g\right] \\
& =\lim _{i \rightarrow \infty} h_{i} \\
& =0
\end{aligned}
$$

whence $\beta \in(f)$, which is impossible because $o(\beta)<a$ (recall that $\beta=f_{0}+\sum_{k=1}^{\infty} f_{k}$, $o\left(f_{0}\right)=a-d$, and $o\left(f_{k}\right) \geq o\left(h_{0}\right)-b>a-d$ for all $\left.k \geq 1\right)$. Thus $(\alpha f+\beta g)^{*} \notin\left(f^{*}, g^{*}\right)$ for some elements $\alpha, \beta$ of $S$ with $o(\alpha)=b-d$ and $o(\beta)=a-d$. 
Remark 2.3. Let $\alpha, \beta \in S$ with $o(\alpha)=b-d$ and assume that $(\alpha f+\beta g)^{*} \notin\left(f^{*}, g^{*}\right)$. Then $\alpha^{*}=-\bar{u} \eta$ and $\beta^{*}=\bar{u} \xi$ for some unit $u$ in $S$. Hence $\alpha^{*}, \beta^{*}$ form a $G$-regular sequence.

Proof. With the same notation as in the proof of Lemma 2.1 we have $0 \neq \varphi \in G_{0}=$ $S / \mathfrak{n}$. Letting $\varphi=\bar{u}$ with a unit $u$ in $S$, we readily get $\alpha^{*}=-\bar{u} \eta$ and $\beta^{*}=\bar{u} \xi$.

Let $n=\mu_{G}(J)$ and $k=S / \mathfrak{n}$. In Proposition 2.4 (3) we prove the uniqueness of the order of $o(\alpha f+\beta g)$ for the elements $\alpha$ and $\beta$ in $S$ given by Proposition 2.2 and the uniqueness of the ideal $\left(f^{*}, g^{*}, h^{*}\right)$ as well, where $h=\alpha f+\beta g$.

Proposition 2.4. Let $\alpha, \beta, \sigma, \tau \in S$ with $o(\alpha)=b-d$. Let $h=\alpha f+\beta g$ and $q=\sigma f+\tau g$. Assume that $h^{*} \notin\left(f^{*}, g^{*}\right)$. Then the following assertions hold true.

(1) Assume that $q^{*} \notin\left(f^{*}, g^{*}\right)$. Then $o(q) \geq o(h)+o(\sigma)-(b-d)$.

(2) Assume that $q^{*} \notin\left(f^{*}, g^{*}, h^{*}\right)$. Then $o(q)>o(h)+o(\sigma)-(b-d)$.

(3) Assume that $q^{*} \notin\left(f^{*}, g^{*}\right)$ and $o(\sigma)=b-d$. Then $o(q)=o(h)$ and $\left(f^{*}, g^{*}, q^{*}\right)=\left(f^{*}, g^{*}, h^{*}\right)$.

(4) The elements $f^{*}, g^{*}, h^{*}$ form a part of a minimal system of homogeneous generators of $J$.

(5) Assume that $n \geq 4$ and $I \subseteq \mathfrak{n}^{2}$. Then writing $J=\bigoplus J_{n}$, we have $J \supsetneq\left(J_{i} \mid 1 \leq i \leq 5\right) G$.

Proof. Assume that $q^{*} \notin\left(f^{*}, g^{*}\right)$ and let $c=o(\sigma)-(b-d)$. Then $\sigma^{*} \xi+\tau^{*} \eta=0$ by Lemma 2.1. Choose a unit $u$ in $S$ so that $\alpha^{*}=-\bar{u} \eta$ and $\beta^{*}=\bar{u} \xi$. Then, since $\sigma^{*} \xi \bar{u}+\tau^{*} \eta \bar{u}=0$, we get $\sigma^{*} \beta^{*}=\tau^{*} \alpha^{*}$. Hence $\sigma^{*}=\alpha^{*} \delta^{*}$ and $\tau^{*}=\beta^{*} \delta^{*}$ for some $\delta \in S$ with $o(\delta)=c$, because $\alpha^{*}, \beta^{*}$ is a $G$-regular sequence. Thus $\sigma=\alpha \delta+\sigma_{1}$ and $\tau=\beta \delta+\tau_{1}$ for some $\sigma_{1}, \tau_{1} \in S$ with $o\left(\sigma_{1}\right)>o(\sigma)$ and $o\left(\tau_{1}\right)>o(\tau) ;$

$$
q=h \delta+\left(\sigma_{1} f+\tau_{1} g\right) .
$$

Now let

$$
\Lambda=\left\{\begin{array}{l|l}
o\left(\sigma^{\prime} f+\tau^{\prime} g\right) \mid \begin{array}{l}
\sigma^{\prime}, \tau^{\prime} \in S \text { such that } \\
\left(\sigma^{\prime} f+\tau^{\prime} g\right)^{*} \notin\left(f^{*}, g^{*}\right) \text { and } o\left(\sigma^{\prime}\right) \geq b-d+c
\end{array}
\end{array} .\right.
$$

Then $o(q) \in \Lambda$. Let $n=\min \Lambda$ and put $\Gamma=\left\{o\left(\sigma^{\prime}\right) \mid \begin{array}{l}\sigma^{\prime} \in S \text { for which there exists } \tau^{\prime} \in S \text { such that } \\ \left(\sigma^{\prime} f+\tau^{\prime} g\right)^{*} \notin\left(f^{*}, g^{*}\right), o\left(\sigma^{\prime}\right) \geq b-d+c, \text { and } o\left(\sigma^{\prime} f+\tau^{\prime} g\right)=n\end{array}\right\}$. 
Then $\Gamma \neq \emptyset$ and $\gamma<n-a$ for all $\gamma \in \Gamma$ (cf. Lemma 2.1 (1)). Let $\gamma=\max \Gamma$ and choose $\sigma^{\prime}, \tau^{\prime} \in S$ so that $\left(\sigma^{\prime} f+\tau^{\prime} g\right)^{*} \notin\left(f^{*}, g^{*}\right), \gamma=o\left(\sigma^{\prime}\right) \geq b-d+c$, and $o\left(\sigma^{\prime} f+\tau^{\prime} g\right)=n$. Let $q^{\prime}=\sigma^{\prime} f+\tau^{\prime} g$. Then, because $q^{\prime *} \notin\left(f^{*}, g^{*}\right)$, similarly as in equation (1) we have

$$
q^{\prime}=h \delta^{\prime}+\left(\sigma_{2} f+\tau_{2} g\right)
$$

for some $\delta^{\prime}, \sigma_{2}, \tau_{2} \in S$ with $o\left(\delta^{\prime}\right)=o\left(\sigma^{\prime}\right)-(b-d), o\left(\sigma_{2}\right)>o\left(\sigma^{\prime}\right)$, and $o\left(\tau_{2}\right)>o\left(\tau^{\prime}\right)$. Let $q^{\prime \prime}=\sigma_{2} f+\tau_{2} g$ and assume that $o\left(q^{\prime}\right)<o\left(h \delta^{\prime}\right)$. We then have

$$
n=o\left(q^{\prime}\right)=o\left(q^{\prime \prime}\right) \quad \text { and } \quad q^{*}=q^{\prime \prime *},
$$

whence $q^{\prime \prime *} \notin\left(f^{*}, g^{*}\right)$. On the other hand, because $o\left(\sigma_{2}\right)>o\left(\sigma^{\prime}\right) \geq b-d+c$, we get $o\left(\sigma_{2}\right) \in \Gamma$, which is impossible (recall that $o\left(\sigma^{\prime}\right)=\max \Gamma$ ). Thus $o\left(q^{\prime}\right) \geq o\left(h \delta^{\prime}\right)$ and so

$$
\begin{aligned}
o(q) & \geq n=o\left(q^{\prime}\right) \geq o(h)+o\left(\delta^{\prime}\right) \\
& =o(h)+o\left(\sigma^{\prime}\right)-(b-d) \\
& \geq o(h)+[(b-d)+c]-(b-d) \\
& =o(h)+c,
\end{aligned}
$$

as was claimed. This proves assertion (1).

Now assume that $q^{*} \notin\left(f^{*}, g^{*}, h^{*}\right)$. Then $o(q) \geq o(h)+c$ by assertion (1), where $c=o(\sigma)-(b-d)$. Assume $o(q)=o(h)+c$ and write $q=h \delta+\left(\sigma_{1} f+\tau_{1} g\right)$ for some $\delta, \sigma_{1}, \tau_{1} \in S$ with $o(\delta)=c, o\left(\sigma_{1}\right)>o(\sigma)$, and $o\left(\tau_{1}\right)>o(\tau)$ (cf. equation (1)). We put $q_{1}=\sigma_{1} f+\tau_{1} g$. Then, because $o(q)=o(h \delta) \geq \min \left\{o(h \delta), o\left(q_{1}\right)\right\}, o\left(q_{1}\right) \geq o(h \delta)$. If $o\left(q_{1}\right)>o(h \delta)$, then we have $q^{*}=(h \delta)^{*}=h^{*} \delta^{*} \in\left(f^{*}, g^{*}, h^{*}\right)$, which is impossible. Hence $o\left(q_{1}\right)=o(h \delta)=o(q)$ so that $q^{*}=h^{*} \delta^{*}+q_{1}^{*} \notin\left(f^{*}, g^{*}, h^{*}\right)$. Consequently $q_{1}^{*} \notin\left(f^{*}, g^{*}\right)$ and so we get by assertion (1) that

$$
\begin{aligned}
o(h)+c & =o(h \delta)=o\left(q_{1}\right) \\
& \geq o(h)+o\left(\sigma_{1}\right)-(b-d) \\
& \geq o(h)+[o(\sigma)+1]-(b-d) \\
& =o(h)+c+1,
\end{aligned}
$$

which is absurd. Hence $o(q)>o(h)+c$. This proves assertion (2). 
To show assertion (3), thanks to assertion (2), it is enough to check the equality $o(q)=o(h)$. The inequality $o(q) \geq o(h)$ follows from assertion (1), whence $o(h)=$ $o(q)$ by symmetry.

We now prove assertions (4) and (5). Let $V=J / N J$ and choose homogeneous elements $\delta_{1}, \delta_{2}, \ldots, \delta_{n}$ of $J$ so that their images $\overline{\delta_{1}}, \overline{\delta_{2}}, \ldots, \overline{\delta_{n}}$ in $V$ form a $k$-basis of $V$. We may assume $\delta_{1}=f^{*}, \delta_{2}=g^{*}$. Hence $J=\left(f^{*}, g^{*}, \delta_{3}, \ldots, \delta_{n}\right)$. For each $3 \leq i \leq n$ let $\delta_{i}=q_{i}^{*}$ with $q_{i} \in I$ and write $q_{i}=\sigma_{i} f+\tau_{i} g$ for some $\sigma_{i}, \tau_{i} \in S$. Then $o\left(\sigma_{i}\right) \geq b-d$ by Lemma 2.1. We have $o\left(q_{i}\right)=o(h)$ and $\left(f^{*}, g^{*}, q_{i}^{*}\right)=\left(f^{*}, g^{*}, h^{*}\right)$ $\left(\right.$ resp. $\left.o\left(q_{i}\right)>o(h)\right)$, if $o\left(\sigma_{i}\right)=b-d$ (resp. if $o\left(\sigma_{i}\right)>b-d$ ) by assertion (3) (resp. assertion (1)). Hence $o\left(q_{i}\right) \geq o(h)$. We may assume $o\left(q_{3}\right) \leq o\left(q_{4}\right) \leq \ldots \leq o\left(q_{n}\right)$. Then, because $h^{*} \in\left(f^{*}, g^{*}, \delta_{3}, \delta_{4}, \ldots, \delta_{n}\right)$ but $h^{*} \notin\left(f^{*}, g^{*}\right)$, we get $\operatorname{deg} h^{*}=o(h) \geq$ $\operatorname{deg} \delta_{3}=o\left(q_{3}\right)$ so that $o\left(q_{3}\right)=o(h)$, whence $\left(f^{*}, g^{*}, \delta_{3}\right)=\left(f^{*}, g^{*}, h^{*}\right)$ by assertion (3). Thus assertion (4) follows. Suppose that $n \geq 4$. Then $\delta_{4}=q_{4}^{*} \notin\left(f^{*}, g^{*}, \delta_{3}\right)=$ $\left(f^{*}, g^{*}, h^{*}\right)$. Therefore $o\left(\sigma_{4}\right)>b-d$. Hence by assertion (2) we have

$$
\begin{aligned}
\operatorname{deg} \delta_{4} & =o\left(q_{4}\right) \\
& \geq o(h)+\left[o\left(\sigma_{4}\right)-(b-d)+1\right] \geq o(h)+2 \\
& \geq(a+b-d)+3 \\
& \geq b+4 \geq a+4 .
\end{aligned}
$$

Consequently, $\operatorname{deg} \delta_{4}=o\left(q_{4}\right) \geq 6$, if $I \subseteq \mathfrak{n}^{2}$. Hence $J \supsetneq\left(J_{i} \mid 1 \leq i \leq 5\right) G$, which completes the proof of Proposition 2.4.

We are now ready to prove Theorem 1.2 .

Proof of Theorem 1.2. We may assume that $S$ is complete and $h_{G}\left(f^{*}, g^{*}\right)=1$. Hence $\mu_{G}(J)=3$. Choose $\alpha, \beta \in S$ so that $o(\alpha)=b-d$ and $(\alpha f+\beta g)^{*} \notin\left(f^{*}, g^{*}\right)$. Let $h=\alpha f+\beta g$. Then $J=\left(f^{*}, g^{*}, h^{*}\right)$ by Proposition 2.4 (4). We furthermore have $h^{*} \in\left(\alpha^{*}, \beta^{*}\right)$, because $\alpha^{*}, \beta^{*}$ is a $G$-regular sequence (cf. Remark 2.3) and $h \in(\alpha, \beta)$. Let $h^{*}=\alpha^{*} \varphi+\beta^{*} \psi$ with $\varphi, \psi \in G$. Then, since $\alpha^{*}=-\bar{u} \eta$ and $\beta^{*}=\bar{u} \xi$ for some unit $u$ in $S$, we see

$$
J=I_{2}\left(\begin{array}{ccc}
\bar{u} \varphi & \bar{u} \psi & D \\
\xi & \eta & 0
\end{array}\right)
$$

where $D \in G$ is the element such that $f^{*}=\xi D$ and $g^{*}=\eta D$. Thus $J$ is a perfect ideal of $G$, because $\operatorname{grade}_{G} J=2$. 
Discussion 2.5. Assume notation as in Setting 1.1 and also assume that $I \subset \mathfrak{n}^{2}$. Let $\mu\left(I^{*}\right)$ denote the minimal number of generators of $I^{*}$. If $\mu\left(I^{*}\right)=3$, then $I^{*}=\left(f^{*}, g^{*}, h_{0}^{*}\right) G$, where $h_{0}=\alpha f+\beta g$ and $o(\alpha)=b-d$. We have

$$
2 \leq \operatorname{deg} f^{*} \leq \operatorname{deg} g^{*}<\operatorname{deg} g^{*}+2 \leq \operatorname{deg} h_{0}^{*}
$$

so $\operatorname{deg} h_{0}^{*} \geq 4$. If $\mu\left(I^{*}\right) \geq 4$, then there exist homogeneous generators for $I^{*}$ so that

$$
I^{*}=\left(f^{*}, g^{*}, h_{0}^{*}, h_{1}^{*}, \ldots, h_{r}^{*}\right) G
$$

where we have $r=\mu\left(I^{*}\right)-3$, and

$$
2 \leq \operatorname{deg} f^{*} \leq \operatorname{deg} g^{*}<\operatorname{deg} g^{*}+2 \leq \operatorname{deg} h_{0}^{*}<\operatorname{deg} h_{0}^{*}+2 \leq \operatorname{deg} h_{1}^{*} \leq \cdots \leq \operatorname{deg} h_{r}^{*} .
$$

The inequality $\operatorname{deg} h_{1}^{*} \geq \operatorname{deg} h_{0}^{*}+2$ is by Proposition 2.4 (2). In particular, if $\mu\left(I^{*}\right) \geq 4$, then the relation type of $\operatorname{gr}_{\mathfrak{m}}(R)$ is greater than or equal to 6 .

It would be interesting to know whether $\operatorname{deg} h_{i}^{*}+2 \leq \operatorname{deg} h_{i+1}^{*}$ holds for all $i$ with $0 \leq i<r$, or, if this fails to hold in general, whether $\operatorname{deg} h_{i}^{*}+1 \leq \operatorname{deg} h_{i+1}^{*}$. An interesting result of Kothari $[\mathrm{K}]$ shows that if $S$ is a 2-dimensional regular local ring containing a coefficient field, then $\operatorname{deg} h_{i}^{*}+1 \leq \operatorname{deg} h_{i+1}^{*}$ for all $i$ with $1 \leq i<r$.

\section{Applications of the theorem}

Let us give some consequences of Theorem 1.2. We begin with the following.

Corollary 3.1. Let $(R, \mathfrak{m})$ be a d-dimensional Gorenstein local ring. Assume that $\mathfrak{m}$ is minimally generated by $d+2$ elements. Then $\operatorname{gr}_{\mathfrak{m}}(R)$ is a Cohen-Macaulay ring, if the relation type of $\operatorname{gr}_{\mathfrak{m}}(R)$ is less than or equal to 5 .

Proof. We may assume that $(R, \mathfrak{m})$ is complete. Hence, thanks to the structure theorem of Cohen ([BH, Theorem A.21]), we get $R=S / I$, where $I$ is an ideal of a $(d+2)$-dimensional regular local ring $(S, \mathfrak{n})$. Because $R$ is a Gorenstein ring and $\operatorname{dim} R=d$, the ideal $I$ is generated by a regular sequence $f, g$ of length 2 . Let $J=\operatorname{Ker}\left(\operatorname{gr}_{\mathfrak{n}}(S) \stackrel{\varphi}{\rightarrow} \operatorname{gr}_{\mathfrak{m}}(R)\right)$, where $\varphi: \operatorname{gr}_{n}(S) \rightarrow \operatorname{gr}_{m}(R)$ denotes the canonical map. We may assume that $\mu_{\operatorname{gr}_{n}(S)}(J) \geq 3$. Then by Proposition $2.4(5)$ the ideal $J$ is 3 -generated, because the relation type of $\operatorname{gr}_{\mathfrak{m}}(R)$ is at most 5 , whence by Theorem $1.2, \operatorname{gr}_{\mathfrak{m}}(R)$ is a Cohen-Macaulay ring since the polynomial ring $\operatorname{gr}_{\mathfrak{n}}(S)$ is a UFD. 
Corollary 3.2. Let $(R, \mathfrak{m})$ be a one-dimensional Gorenstein local ring and assume that $\mathfrak{m}$ is minimally generated by 3 elements. If the reduction number of $\mathfrak{m}$ is less than or equal to 4 , then $\operatorname{gr}_{\mathfrak{m}}(R)$ is a Cohen-Macaulay ring.

Proof. The result of Huckaba [H, Theorem 2.3] shows that in our setting the relation type of $\operatorname{gr}_{\mathfrak{m}}(R)$ is at most one more than the reduction number of $\mathfrak{m}$. Hence by Corollary 3.1 the ring $\operatorname{gr}_{\mathfrak{m}}(R)$ is Cohen-Macaulay.

The example studied in Example 1.6 shows that Corollary 3.2 may fail if the reduction number of $\mathfrak{m}$ is 5 . The following example is explored by Sally [S, Example 2.2] and shows that Corollary 3.1 may fail if we assume that $R$ is a Cohen-Macaulay (rather than Gorenstein) ring.

Example 3.3. Let $S=k[[x, y, z]]$ be the formal power series ring with three variables $x, y, z$ over a field $k$. Let $R=k\left[\left[w^{4}, w^{5}, w^{11}\right]\right]$ be the subring of the formal power series ring $k[[w]]$ and consider the homomorphism $\phi: S \rightarrow R$ of $k$-algebras defined by $\phi(x)=w^{4}, \phi(y)=w^{5}$, and $\phi(z)=w^{11}$. Then $I=\operatorname{Ker} \phi$ is generated by $x z-y^{3}, y z-x^{4}$, and $z^{2}-x^{3} y^{2}$. We have $\operatorname{gr}_{\mathfrak{n}}(S)=k\left[x^{*}, y^{*}, z^{*}\right]$,

$$
I^{*}=\left(z^{* 2}, z^{*} y^{*}, z^{*} x^{*}, y^{* 4}\right),
$$

and the ring $\operatorname{gr}_{\mathfrak{m}}(R)=\operatorname{gr}_{\mathfrak{n}}(S) / I^{*}$ is not Cohen-Macaulay. The relation type of $\operatorname{gr}_{\mathfrak{m}}(R)$ is 4 and the reduction number of $\mathfrak{m}$ is 3 .

Corollary 3.4. Let $(R, \mathfrak{m})$ be a one-dimensional Gorenstein local ring and assume that $\mathfrak{m}$ is minimally generated by 3 elements. If the reduction number $r$ of $\mathfrak{m}$ is less than or equal to 4 , then $\operatorname{gr}_{\mathfrak{m}}(R)$ is a Gorenstein ring if and only if $J^{r}: \mathfrak{m}^{r}=\mathfrak{m}^{r}$, where $J$ is a reduction of $\mathfrak{m}$.

Proof. By Corollary 3.2, $\operatorname{gr}_{\mathfrak{m}}(R)$ is Cohen-Macaulay. Therefore all the powers of $\mathfrak{m}$ are closed in the sense of Ratliff-Rush. Hence $\operatorname{gr}_{\mathfrak{m}}(R)$ is a Gorenstein ring if and only if $J^{r}: \mathfrak{m}^{r}=\mathfrak{m}^{r}$ (cf. [HKU, Corollary 4.8]).

Acknowledgement. The main question considered in this paper arose during the collaboration of W. Heinzer and M-K Kim with Bernd Ulrich in [HKU]. We would like to thank him for his help in developing this material. 


\section{REFERENCES}

[BH] W. Bruns and J. Herzog, Cohen-Macaulay Rings, Cambridge University Press, Cambridge, 1993.

[HKU] W. Heinzer, Mee-Kyoung Kim and B. Ulrich, The Gorenstein and complete intersection properties of associated graded rings, J. Pure Appl. Algebra, to appear.

$[\mathrm{H}] \quad$ S. Huckaba, Reduction numbers for ideals of higher analytic spread, Math. Proc. Camb. Phil. Soc. 102(1987), 49-57.

[K] S. Kothari, The local Hilbert function of a pair of plane curves, Proc. Amer. Math. Soc. 72 (1978), 439-442.

[S] J. D. Sally, Tangent cones at Gorenstein singularities, Compositio Math. 40 (1980), 167175.

Department of Mathematics, School of Science and Technology Meiji University, 214-8571 JAPAN

E-mail address: goto@math.meiji.ac.jp

Department of Mathematics, Purdue University, West Lafayette, Indiana 47907

E-mail address: heinzer@math.purdue.edu

Department of Mathematics, Sungkyunkwan University Jangangu Suwon 440-746, KOREA

E-mail address: mkkim@math.skku.ac.kr 